\title{
THE TOPOLOGICAL ENTROPY OF HOMEOMORPHISMS ON ONE-DIMENSIONAL CONTINUA
}

\author{
GERALD T. SEIDLER
}

(Communicated by James E. West)

\begin{abstract}
We show that the topological entropy of every homeomorphism on a regular curve is zero. Also, we show the existence of nonrational curves admitting homeomorphisms with nonzero topological entropy.
\end{abstract}

\section{DEFINITIONS AND PRELIMINARY RESUltS}

Let $\mathbf{A}$ and $\mathbf{B}$ be open covers of a compact metric topological space $X$. The join of $\mathbf{A}$ and $\mathbf{B}$, denoted $\mathbf{A} \vee \mathbf{B}$, is $\{A \cap B \mid A \in \mathbf{A}$ and $B \in \mathbf{B}\}$. Let $N(\mathbf{A})$ denote the number of elements in a subcover of $\mathbf{A}$ of minimum cardinality. An open cover $\mathbf{A}$ refines another open cover $\mathbf{B}$ if every element of $\mathbf{A}$ is contained in some element of $\mathbf{B}$. The mesh of an open cover $\mathbf{A}$ is the supremum of the diameter of the elements of $\mathbf{A}$. A single arrow $\rightarrow$ will be used to indicate that a continuous function is into while a double arrow $\rightarrow$ will denote an onto function.

One-dimensional continua embedded in $E^{n}$ can be placed into three categories depending on the characteristics of their open covers. Let $S$ be a onedimensional continuum in $E^{n}$. If there exist open covers of $S$ of arbitrarily small mesh such that every element of the cover has finite boundary then $S$ is said to be a regular curve. If instead at least one element will have countably infinite boundary but none will ever necessarily have uncountable boundary, then $S$ is said to be a rational curve. Finally, if open covers of arbitrarily small mesh need always eventually have an element with uncountable boundary then we call $S$ a nonrational curve. Recall that every open cover of a compact metric space has a Lebesgue number. Thus, for each regular (rational/nonrational) curve there will exist a refining sequence of minimal open covers such that the total number of boundary points of elements of a given cover is finite (countable/uncountable).

The definition of topological entropy given below and employed here is that of Adler, Konheim, and McAndrew [1]. Let $f: X \rightarrow X$ be continuous and let

Received by the editors July 25,1988 .

1980 Mathematics Subject Classification (1985 Revision). Primary 54C70, 54F20, 54H20.

Key words and phrases. Entropy, regular curves, one-dimensional continua. 
$f^{-1}(\mathbf{A})$ denote the open cover consisting of the inverse image of every element of $\mathbf{A}$; inductively define $f^{-i}$ for all positive integers $i$. Let the topological entropy of $f$ with respect to $\mathbf{A}$, denoted $\operatorname{ent}(f, \mathbf{A})$, be defined by

$$
\operatorname{ent}(f, \mathbf{A})=\lim _{n \rightarrow \infty} n^{-1} \log \left(N\left(\mathbf{A} \vee f^{-1}(\mathbf{A}) \vee \cdots \vee f^{-(n-1)}(\mathbf{A})\right)\right)
$$

where the logarithm is to the base 2 in this paper except where otherwise noted. The topological entropy of $f$, often shortened to the entropy of $f$ and denoted ent $(f)$, is defined to be the supremum of the topological entropies of $f$ with respect to all open covers of $X$. Theorems 1.1 to 1.4 are from [1], while Theorem 1.5 is an extension of a theorem from [1]. The author thanks Dr. Albert Fathi of the University of Florida for pointing out that Theorem 1.5 holds not only for homeomorphisms but rather for all continuous functions $h: X \rightarrow X$ and $g: Y \rightarrow Y$.

Theorem 1.1. Let $\left\{\mathbf{A}_{n}\right\}$ be a refining sequence of open covers of a compact metric space $X$ such that their mesh converges to zero. Then

$$
\operatorname{ent}(f)=\lim _{n \rightarrow \infty} \operatorname{ent}\left(f, \mathbf{A}_{n}\right) .
$$

Theorem 1.2. Let $X$ and $Y$ be compact topological spaces and let $f: X \rightarrow X$ and $g: Y \rightarrow Y$ be continuous functions into $X$ and $Y$, respectively. Then $\operatorname{ent}(f \times g)=\operatorname{ent}(f)+\operatorname{ent}(g)$ where $f \times g$ is the continuous function defined by

$$
f \times g:(x, y) \mapsto(f(x), g(y)) .
$$

Theorem 1.3. Let $X_{1}$ and $X_{2}$ be closed subsets of a compact topological space $X$ such that $X=X_{1} \cup X_{2}$ and $f\left(X_{1}\right) \subseteq X_{1}$ and $f\left(X_{2}\right) \subseteq X_{2}$ for some continuous function $f: X \rightarrow X$. Then $\operatorname{ent}(f)=\max \left\{\operatorname{ent}\left(f \mid X_{1}\right)\right.$, ent $\left.\left(f \mid X_{2}\right)\right\}$ where $f \mid X_{1}$ or $X_{2}$ denotes the restriction of $f$ to $X_{1}$ or $X_{2}$, respectively.

Theorem 1.4. Let $Y=\{0,1, \ldots, N-1\}$ for some fixed positive integer $N$, and let $C_{N}=\prod_{i=-\infty}^{+\infty} Y_{i}$ where each $Y_{1}=Y$. Note that $C_{N}$ is topologically a Cantor set. Let $x \in C_{N}$ so that $x=\left\{x_{i}\right\}$. The shift map s: $C_{N} \rightarrow C_{N}$ is defined by $(s(x))_{i}=x_{i+1} \quad\left(s\right.$ can easily be shown to be a homeomorphism onto $\left.C_{N}\right)$. Then $\operatorname{ent}(s)=\log (N)$.

Theorem 1.5. Let $X$ and $Y$ be compact metric spaces and let $f: X \rightarrow Y$ be continuous and onto. If there exist continuous functions $g: X \rightarrow X$ and $h: Y \rightarrow$ $Y$ into $X$ and $Y$, respectively, such that the diagram in Figure 1 commutes, then $\operatorname{ent}(g) \geq \operatorname{ent}(h)$.

Proof. Let $\left\{\mathbf{A}_{n}\right\}$ be a refining sequence of minimal open covers of $Y$ such that their mesh converges to zero. Then $\left\{f^{-1}\left(\mathbf{A}_{n}\right)\right\}$ is a refining sequence of minimal open covers of $X$, but whose mesh may not converge to zero because $f$ may not be one-to-one. The diagram in Figure 1 commuting gives $h \cdot f=f \cdot g$, 


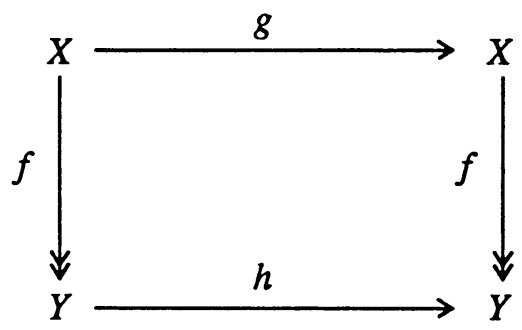

Figure 1.

so that $f \cdot g^{-1}=h^{-1} \cdot f$ and thus $f \cdot g^{-n}=h^{-n} \cdot f$ by induction on $n$. Let A be an element of $\left\{\mathbf{A}_{n}\right\}$. Then

$$
\begin{aligned}
\operatorname{ent}\left(g, f^{-1}(\mathbf{A})\right) & =\lim _{n \rightarrow \infty} n^{-1} \log \left(N\left(\bigvee_{i=0}^{n-1} g^{-i}\left(f^{-1}(\mathbf{A})\right)\right)\right) \\
& =\lim _{n \rightarrow \infty} n^{-1} \log \left(N\left(\bigvee_{i=0}^{n-1} f^{-1}\left(h^{-i}(\mathbf{A})\right)\right)\right) \\
& \geq \lim _{n \rightarrow \infty} n^{-1} \log \left(N\left(\bigvee_{i=0}^{n-1}\left(h^{-i}(\mathbf{A})\right)\right)\right) \\
& =\operatorname{ent}(h, \mathbf{A}) .
\end{aligned}
$$

Thus for each positive integer $n$,

$$
\operatorname{ent}\left(g, f^{-1}\left(\mathbf{A}_{n}\right)\right) \geq \operatorname{ent}\left(h, \mathbf{A}_{n}\right)
$$

so that

$$
\operatorname{ent}(g) \geq \sup \left\{\operatorname{ent}\left(g, f^{-1}\left(\mathbf{A}_{n}\right)\right)\right\} \geq \lim _{n \rightarrow \infty} \operatorname{ent}\left(h, \mathbf{A}_{n}\right)=\operatorname{ent}(h) .
$$

\section{REGULAR CURVES}

The major result of this section is Theorem 2.3 which shows that the entropy of a homeomorphism on a regular curve is zero. It has been shown that the entropy of a homeomorphism on a circle is zero [1], from which it can easily be shown that the entropy of a homeomorphism on the closed interval is zero by identifying the end points of the interval and considering the entropy of the corresponding homeomorphism on a circle.

Lemma 2.1. Let $\mathbf{A}$ be an open cover of a continuum $X$ and let $\mathbf{P}$ be the set of boundary points of elements of $\mathbf{A}$. If $\mathbf{A}$ is a minimal cover then the number of elements of $\mathbf{A}$ is at most the number of elements in $\mathbf{P}$.

Proof. Assume that $\mathbf{A}$ does not have a proper subcover. Then $\mathbf{A}$ is a finite collection because $X$ is compact. Let $a$ be the number of elements of $\mathbf{A}$ so 
that $\mathbf{A}=\left\{A_{i}\right\}$ such that $1 \leq i \leq a$. Let $A_{j} \in \mathbf{A}$, and let $V=\left(\bigcup_{i \neq j} A_{i}\right)$. Note that $V$ is open since it is a union of open sets. Because A doesn't have a proper subcover we see that $X-V \neq \varnothing$ so that $\operatorname{Bd}(V) \neq \varnothing$, since $X$ is connected. Further $\operatorname{Bd}(V) \subseteq \mathbf{P}$ because $V$ is a finite union of elements of $\mathbf{A} . V$ open requires $\operatorname{Bd}(V) \cap V=\varnothing$ so that $\operatorname{Bd}(V) \subseteq A_{j}$. Thus for each element $A_{j}$ of $\mathbf{A}$, there must exist a nonempty subset of $\mathbf{P}$ contained in $A_{j}$ but disjoint from every other element of $\mathbf{A}$. This requires that the number of elements of $\mathbf{A}$ be at most the number of elements in $\mathbf{P}$.

Lemma 2.2. Let $h: S \rightarrow S$ be a homeomorphism of a regular curve $S$ into itself. Let A be a minimal open cover of $S$ of at least two elements such that every element of $\mathbf{A}$ has finite boundary. Let $L_{A}$ be the total number of boundary points of elements of $\mathbf{A}$. For each positive integer $n$, let $\mathbf{M}_{n}$ be a subcover of minimum cardinality of $\bigvee_{i=0}^{n-1} h^{-i}(\mathbf{A})$ and let $\mathbf{P}_{n}$ be the collection of boundary points of elements of $\bigcup_{i=0}^{n-1} h^{-i}(\mathbf{A})$. Then

(i) For each positive integer $n$ every boundary point of an element of $\mathbf{M}_{n}$ is in $\mathbf{P}_{n}$.

(ii) $N\left(\bigvee_{i=0}^{n-1} h^{-i}(\mathbf{A})\right) \leq n L_{A}$.

Proof of Part (i). Let $n$ be a positive integer and let $x$ be a boundary point of $M \in \mathbf{M}_{n}$. By the definition of $\mathbf{M}_{n}$, for each positive integer $i \leq n$, there exists $V_{i} \in \mathbf{M}_{i}$ such that $M=\bigcap_{i=1}^{n-1} V_{i}$. Let $x$ be a boundary point of $M$ and let $B$ be an open set containing $x$; clearly $B \cap M \neq \varnothing$ so that $B \cap V_{i} \neq \varnothing$ for each $V_{i}$. For each $V_{i}$, this requires that either $x \in \operatorname{Bd}\left(V_{i}\right)$ or $x \in V_{i}$. Suppose every $V_{i}$ contains $x$. Then $x \in M$ by definition. But this contradicts $x \in \operatorname{Bd}(M)$, because $M$ is open. Thus $x$ is a boundary point of at least one $V_{i}$ and thus $x \in \mathbf{P}_{n}$.

Proof of Part (ii). Let $n$ be a positive integer. Because $h$ is a homeomorphism the number of boundary points of elements of $h^{j}(\mathbf{A})$ is $L_{A}$ for every integer $j$. This requires that the number of elements of $\mathbf{P}_{n}$ be at most $n L_{A}$. As every boundary point of an element of $\mathbf{M}_{n}$ is in $\mathbf{P}_{n}$ and as $\mathbf{M}_{n}$ doesn't have a proper subcover, Lemma 2.1 implies there exist at most $n L_{A}$ elements in $\mathbf{M}_{n}$. The desired result then follows from the definition of $\mathbf{M}_{n}$.

Theorem 2.3. Every homeomorphism on a regular curve has zero topological entropy.

Proof. Let $S$ be a regular curve and let $\mathbf{A}$ be a minimal open cover of $S$ containinig at least two elements such that every element of $\mathbf{A}$ has finite boundary. Let $L_{A}$ be the total number of boundary poiints of elements of $\mathbf{A}$. Let $h: S \rightarrow S$ be a homeomorphism of $S$ into itself. We have then from Part (ii) of Lemma 
2.2 that

$$
\begin{aligned}
\operatorname{ent}(h, \mathbf{A}) & =\lim _{n \rightarrow \infty} n^{-1} \log \left(N\left(\bigvee_{i=0}^{n-1} h^{-1}(\mathbf{A})\right)\right) \\
& \leq \lim _{n \rightarrow \infty} n^{-1} \log \left(n L_{A}\right) \\
& =\lim _{n \rightarrow \infty}\left(\log _{e}(2)\right)\left(L_{A} / n L_{A}\right) / 1 \\
& =0,
\end{aligned}
$$

where L'Hôpital's rule has been used. As $\mathbf{A}$ was an arbitrarily chosen minimal open cover of at least two elements such that every element of $\mathbf{A}$ has finite boundary, we see that the entropy of $h$ with respect to every such cover is zero. As $S$ is regular such covers exist with arbitrarily small mesh, and a refining sequence of such covers must exist that satisfy the conditions of Theorem 1.1; thus $\operatorname{ent}(h)=0$.

\section{RATIONAL AND NONRATIONAL CURVES}

In view of the result of Theorem 2.3, the question arises as to whether there are any rational or nonrational curves which allow homeomorphisms with nonzero topological entropy. We prove a sufficient (but possibly not necessary) condition in Theorem 3.1 for the existence of rational and nonrational curves admitting homeomorphisms with nonzero topological entropy. We observe in Example 3.2 that the sufficient conditions for a nonrational curve to exhibit a homeomorphism with nonzero topological entropy are satisfied. Conjectures 3.3 and 3.4 deal with rational curves. Note that Conjecture 3.4 implies Conjecture 3.3 .

Theorem 3.1. Let $X$ be a compact zero-dimensional countably (uncountably) infinite point set. Let $h: X \rightarrow X$ be a homeomorphism of $X$ into itself. Then there exists a rational (nonrational) curve $F_{x}$ which admits a homeomorphism with the same topoplogical entropy as $h$.

Proof. By Theorem 1.2 the topological entropy of $h \times$ Id: $X \times I \rightarrow X \times I$, where $I$ is the closed unit interval, will be ent $(h)$. Let $F_{x}$ be the quotient space formed by identifying all the points of the form $(x, 1)$, i.e. $F_{x}$ is the cone over $X$ (see Figure 2). Then there must exist a continuous function $f: X \times I \rightarrow F_{x}$ which performs the desired identification of endpoints and preserves the second coordinate along the intervals that make up the "edges" of the cone. As the inverse image under $f$ of the vertex of the cone $F_{x}$ is invariant under $h$, we see that $g=f \cdot h \cdot f^{-1}: F_{x} \rightarrow F_{x}$ is a homeomorphism. The free endpoints of $F_{x}$ are clearly homeomorphic to the original closed point set $X$ so that the restriction of $g$ to this closed subset of $F_{x}$ is ent $(h)$. Then ent $(g) \geq \operatorname{ent}(h)$ by Theorem 1.3. By the definition of $g$ we have $g \cdot f=f \cdot h$ so that Theorem 1.5 gives $\operatorname{ent}(g) \leq \operatorname{ent}(h)$, and thus $\operatorname{ent}(g)=\operatorname{ent}(h)$. As $X$ is countably (uncountably) infinite the boundary of a disc neighborhood of radius one-half 


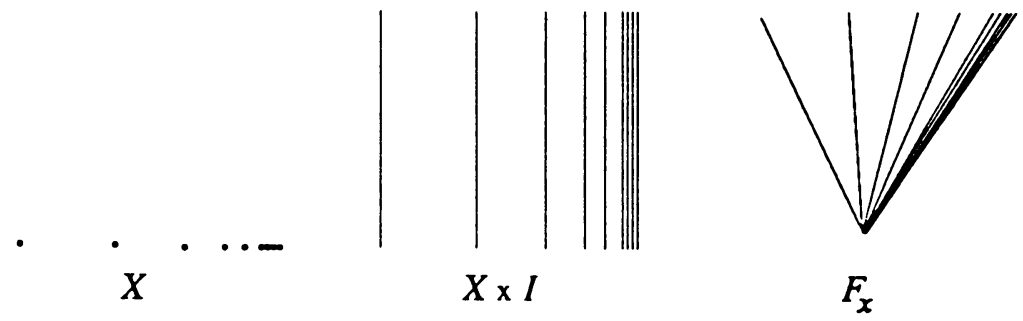

FIGURE 2.

centered at the vertex of $F_{x}$ will have countable (uncountable) boundary, so that $F_{x}$ must be a rational (nonrational) curve.

Example 3.2. Recall that the countable product of a finite set with itself is a Cantor set and thus uncountable. From Theorem 1.4 we know that for each $N$ there exists a Cantor set $C_{N}$ (which can be embedded in a closed region of the plane) such the shift map $s$ on $C_{N}$ is a homeomorphism and has entropy $\log (N)$. Thus the cone over $C_{N}$, also called the Cantor Fan, defined as in the proof to Theorem 3.1 is a nonrational curve such that $s \times \mathrm{Id}$ is a homeomorphism onto it with entropy $\log (N)$.

Conjecture 3.3. For each $N, C_{N}$ cannot be imbedded in any rational curve $X$ in such a way that the shift map $s: C_{n} \rightarrow C_{N}$ can be extended to a homeomorphism on $X$.

Conjecture 3.4. Every homeomorphism on a rational curve has zero topological entropy.

\section{ACKNOWLEDGMENT}

The author expresses his gratitude to Dr. Beverly Brechner of the University of Florida for many useful discussions and suggestions during the development and preparation of this work.

\section{BIBLIOGRAPHY}

1. R. L. Adler, A. G. Konheim, and M. H. McAndrew, Topological entropy, Trans. Amer. Math. Soc. 114 (1965), 309-319.

Department of Mathematics, University of Florida, Gainesville, Florida 32610

Current address: The James Franck Institute and the Department of Physics, University of Chicago, 5640 S. Ellis Avenue, Chicago, Illinois 60637 